\title{
One-dimensional acoustic potential landscapes guide the neurite outgrowth and affect the viability of B35 neuroblastoma cells
}

Kathrin Baumgartner

Sophie Mauritz

Sebastian Angermann

Manuel Brugger

Christoph Westerhausen ( $\nabla$ christoph.westerhausen@gmail.com )

University of Augsburg https://orcid.org/0000-0001-7103-7060

\section{Article}

Keywords: B35 Cells, Acoustic Tweezers, Neurite Outgrowth, Surface Acoustic Waves, Cell Viability, Proliferation

Posted Date: March 1st, 2022

DOI: https://doi.org/10.21203/rs.3.rs-1370278/v1

License: (c) (1) This work is licensed under a Creative Commons Attribution 4.0 International License.

Read Full License 


\title{
One-dimensional acoustic potential landscapes guide the neurite outgrowth and affect the viability of B35 neuroblastoma cells
}

\author{
Kathrin Baumgartner ${ }^{1,2,3}$, Sophie C. F. Mauritz ${ }^{4}$, Sebastian Angermann ${ }^{4}$, Manuel S. Brugger ${ }^{3,4,5^{*}}$ and Christoph \\ Westerhausen $^{1,3,6^{*}}$ \\ ${ }^{1}$ Physiology, Institute of Theoretical Medicine, University of Augsburg, 86159 Augsburg, Germany \\ ${ }^{2}$ Hanns-Seidel-Stiftung e.V., 80636 Munich, Germany \\ ${ }^{3}$ Center for NanoScience (CeNS), Ludwig-Maximilians-Universität Munich, 80799 Munich, Germany \\ ${ }^{4}$ Institute of Physics, Experimental Physics I, University of Augsburg, 86159 Augsburg, Germany \\ ${ }^{5}$ Stiftung der deutschen Wirtschaft (sdw) gGmbH, 10178 Berlin \\ ${ }^{6}$ Augsburg Center for Innovative Technologies (ACIT), 86159 Augsburg, Germany
}

* co-corresponding authors: brugger.manuel@kabelmail.de and christoph.westerhausen@gmail.com

\begin{abstract}
On the way towards neuronal stimulation and signalling, standing surface acoustic waves (SSAW) have become a widely used technique to create well-defined networks of living cells in vitro during the past years. An overall challenge in this research area is to maintain cell viability in long-term treatments long enough to observe changes in cellular functions. To close this gap, we here investigate SSAWdirected neurite outgrowth of $\mathrm{B} 35$ (neuroblastoma) cells in microchannels on $\mathrm{LiNbO}_{3}$ chips, employing one-dimensional pulsed and continuous $\mathrm{MHz}$-order SSAW signals at different intensities for up to 40 hours. To increase the efficiency of future investigations, we explore the limits of applicable SSAW parameters by quantifying their viability and proliferation behaviour in this long-term setup. While cell viability is impaired for power levels above $15 \mathrm{dBm}$, our investigations on SSAW-directed neurite outgrowth reveal a significant increase of neurites growing in preferential directions by up to $31.3 \%$ after 30 hours of SSAW treatment.
\end{abstract}

Key words: B35 Cells, Acoustic Tweezers, Neurite Outgrowth, Surface Acoustic Waves, Cell Viability, Proliferation 


\section{INTRODUCTION}

The controllable formation of neuronal cellular networks in vitro is a crucial first step towards investigations on neuronal signalling behaviour, cell-cell interactions and neuronal stimulation ${ }^{1-3}$. Previous studies proved that surface acoustic waves (SAW) and the acoustic tweezers technique are a powerful tool in this area: The combination of standing SAW (SSAW) and microfluidic techniques enables the patterning of living cells and even primary neurons in well-defined structures on a chip ${ }^{1,4-}$ ${ }^{6}$. Furthermore, it was shown that short-term exhibition of cells to external mechanical forces, applied by an ultrasonic standing-wave field ${ }^{7}$ or atomic force microscopy ${ }^{8}$, can affect cellular processes such as proliferation and apoptosis ${ }^{9}$. Recently, also human mesenchymal stem cells have been stimulated employing high-frequency ( $\mathrm{MHz}$ ) SAW to differentiate towards an osteoblast lineage ${ }^{10}$.

Despite the promising results in this field, the effects of SSAW on cell differentiation and viability in acoustic potential landscapes during long-term incubation and the applicable range of SAW power levels to living cells still lack detailed quantification. These details are crucial for the optimization of experimental approaches towards well-defined neuronal structures and the understanding of SSAWdirected neurite growth. Moreover, this is a challenge for any long-term treatment of living cells in acoustofluidic potential landscapes.

Along these lines, we here investigate a broad range of SSAW power levels and signal types - pulsed and continuous SSAW - and their ability to guide neurite outgrowth of B35 neuroblastoma cells in a microchannel on $\mathrm{LiNbO}_{3}$ chips. The $\mathrm{B} 35$ cell line is a popular model for in vitro studies of neuronal morphology because its differentiation is easy to control and does not require organ donation. Our experiments reveal that the here employed range of SSAW parameters at a frequency $f=71 \mathrm{MHz}$ can promote neurite outgrowth along preferential directions in one-dimensional (1D) acoustic potential landscapes by up to $31.3 \%$. To fill the above-mentioned gap fully, we also provide a detailed study of cell viability employing different SSAW parameters. We show that high-intensity SSAW treatments $\left(P_{\text {in }}\right.$ $\geq 15 \mathrm{dBm}$ ) and high pulse width modulations (PWM) lead to reduced proliferation and to premature cell death compared to low-intensity treatments and low PWM. Moreover, we identify acoustic forces as the main driving factor behind these effects.

\section{RESULTS}

The application of a 1D standing wave field in a customized setup, as shown in Figure $\mathbf{1}$ a, allows us to quantify the effects of different SSAW power levels $P_{\text {in }}$ and pulse width modulations PWM on B35 neurite outgrowth and cell viability. The four interdigital transducers (IDTs) enclose an active area, where the standing surface acoustic wave field is generated. Here, the cells were patterned in the nodes of the SSAW and then incubated at $37{ }^{\circ} \mathrm{C}$ and $5 \% \mathrm{CO}_{2}$ to ensure stable and equal conditions (temperature, $\mathrm{pH}$ ) prior to the experiment.

\subsection{Characterization of the setup: Acoustic forces and temperature}

To determine the acoustic force acting on objects in a pressure maximum of an SSAW of the power $P_{\text {in }}$ in our setup, we tracked the velocity of $10 \mu \mathrm{m}$ polystyrene beads (Polybead ${ }^{\circledR}$-Carboxyl, Polysciences Inc., Warrington, PA, USA) in water moving towards a pressure node after turning on the SSAW (see SI 1). We observed that the velocity reaches a maximum before the beads reach the node of the SSAW. Here, acceleration becomes zero $(a=0)$, which allows us to assume that the maximum acoustic force equals the frictional force given by Stoke's law: $F_{\mathrm{r}}=F_{\mathrm{R}}=6 \pi \eta r v_{\max }$, where $\eta=1 \mathrm{mPas}$ and $r$ the radius of 
the object. We found that the acoustic force as a function of SSAW power follows a linear trend with slope $m=8.32 \mathrm{nN} / \mathrm{mW}$, as displayed in Figure $1 \mathrm{~b}$. To account for the spatial dependence of $F_{\mathrm{R}}$ because of the shape of the potential landscape, we additionally introduce a correction factor of $\sin (2 k x)$ with $x$ as the distance of the beads from the node of the SSAW.

a i)

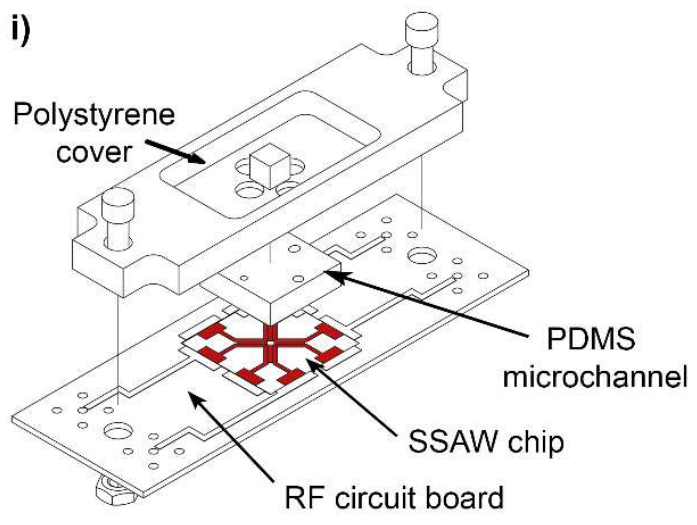

ii)

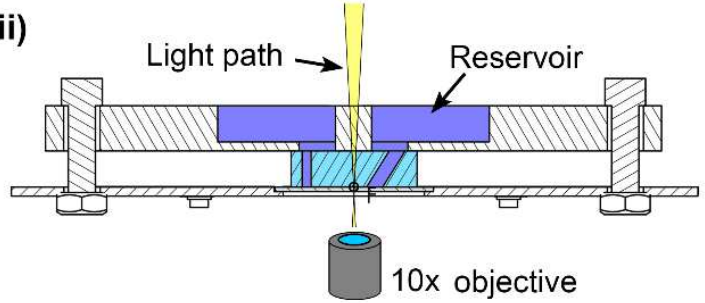

iii)

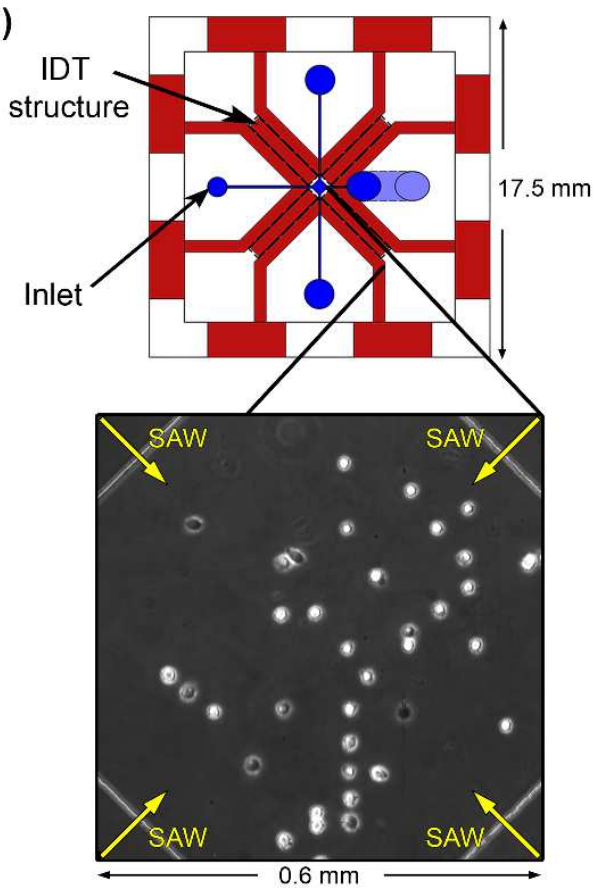

c

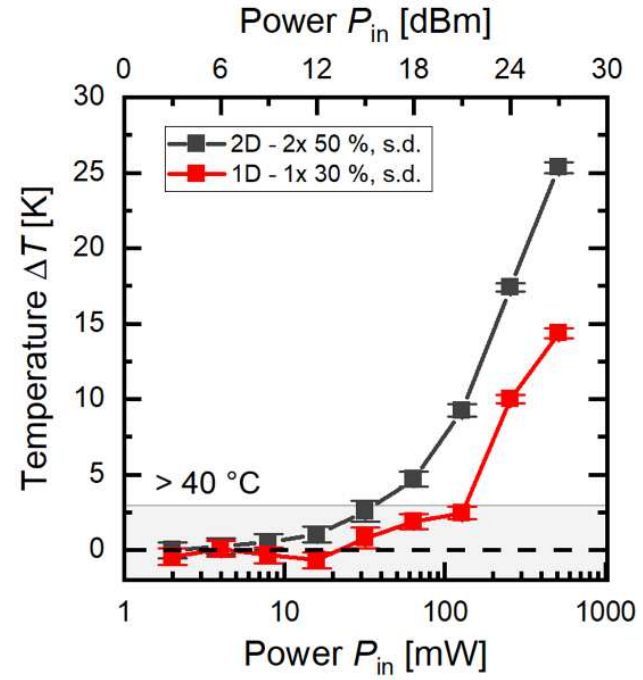

Figure 1: Force field characterization. a) Exploded view (i), side view (ii) and top view of the setup (iii), including a phase contrast image of B35 cells after SSAW patterning in the active area of the chip. b) Acoustic force acting on cells in this setup as a function of applied SSAW power including standard deviations (s.d.). c) Temperature in the setup as a function of applied power for a 2D SSAW at PWM = $50 \%$ and a 1D SSAW at PWM $=30 \%$ (adapted from M. Brugger ${ }^{20}$ ).

As previously described by Kino (1987) $)^{11}$, Yosioka and Kawasima $(1955)^{12}$ and Gor'kov $(1961)^{13}$, the acoustic force acting on an object is given by 


$$
F_{R}(x)=-\left(\frac{\pi V p_{F}^{2} \beta_{F} v}{2 c_{F}}\right) \psi(\beta, \rho) \sin (2 k x)
$$

where $V$ is the volume of the object, $p_{\mathrm{F}}{ }^{2}$ the pressure amplitude, $\beta_{\mathrm{F}}$ the compressibility of the medium, $v=\frac{c_{F}}{\lambda}$ the frequency and $\psi(\beta, \rho)$ the acoustic contrast factor that contains the compressibility $\beta, \beta_{\mathrm{F}}$ and density $\rho, \rho_{F}$ of the object and the medium:

$$
\psi(\beta, \rho)=\frac{5 \rho-2 \rho_{F}}{2 \rho+\rho_{F}}-\frac{\beta}{\beta_{F}} .
$$

The polystyrene beads used here are about the size of a living cell in suspension. By considering the contrast factor of polystyrene $\left(\psi_{\mathrm{PS}}=0.59\right.$ with the materials constants of water and polystyrene $\left.{ }^{14,15}\right)$, (1) can be written as:

$$
F_{R}\left(P_{i n}, \psi\right)=14.03 \frac{n N}{m W} P_{i n} \psi \sin (2 k x)
$$

From here, we are able to calculate the maximum acoustic force acting on cells in our setup by setting $\sin (2 k x)=1$ and inserting a mean contrast factor for cells of $\bar{\psi}_{\text {cell }}=0.2$ (according to literature, contrast factors between 0.14 and 0.25 were found for living cells ${ }^{16}$ ). A well-known side effect of high SAW power levels is the heating of the substrate and medium ${ }^{17}$. The red curve in Figure $1 \mathrm{c}$ shows the temperature development in a microchannel with increasing power of a 1D SSAW at PWM $=30 \%$. We determined the temperature difference by measuring the fluorescence intensity of the thermally sensitive molecule Rhodamine $B^{18,19,20}$. The results show that pulse width and power of the SSAW and therefore the transfer of energy can lead to distinct changes of temperature in the medium.

\subsection{SSAW-guided neurite growth of the B35 neuroblastoma cell line}

To analyse SAW-guided neurite growth, we prepared the samples as described in section 5.1. After cell patterning and adhesion, we added $2 \mathrm{mM}$ DcAMP (dibutyryl cyclic adenosine 3,5-monophosphate) to the medium to induce the differentiation process of the cells. A 1D SSAW at different pulse widths and power levels was employed and the formation of neurites was recorded for $t=40 \mathrm{~h}$. We here test our hypothesis that acoustic forces acting on the cell membrane, filaments and microtubule promote preferred neurite outgrowth along the pressure minima of the one-dimensional acoustic potential landscape.

As illustrated in Figure 2 a, we used the ImageJ plugin NeuronJ ${ }^{21}$ to track the orientation of the neurites. The plugin defines small segments along the neurites with a length $L \approx 5 \mathrm{px}$ (3-4 $\mu \mathrm{m})$. Since, e.g., neurite segments at $\alpha=45^{\circ}$ relative to the $x$ axis appear elongated in the data by a factor of $\sqrt{2}$, we weighted the numbers of segments per class according to the respective angle. In each of the 15 independent experiments, that we analyse in this section, NeuronJ detected about 150 to 2600 neurite segments, which yield an extensive statistic. Next to the phase contrast image, we depict the corresponding relative angle distribution of the neurite segments, divided into 120 classes with a bin width of $3^{\circ}$. After $10 \mathrm{~h}$ of incubation with DcAMP, the neurites in this sample, where no SSAW was employed, do not follow any visible pattern. The histogram confirms that the relative frequencies $P$ in the reference sample are low and equally distributed in all directions. 
On SSAW-treated samples, however, we observed a different tendency: After $10 \mathrm{~h}$, the neurites treated with a $12 \mathrm{dBm}$ continuous SSAW signal (Figure $\mathbf{2}$ b) were more likely to grow along the SSAW pressure minima than in other directions. Additionally, in the sample treated with a $21 \mathrm{dBm}, 30 \%$ pulsed signal (Figure $\mathbf{2} \mathrm{c}$ ), high frequencies of neurite segments do not only appear perpendicular to the SSAW propagation axis but also parallel to it by crossing pressure maxima. An explanation for this effect might be the coupling of SAW into the liquid, which leads to acoustic streaming along the propagation axis of the wave ${ }^{22}$. Neurites following this axis might have an advantage over neurites growing perpendicular to the streaming, because they exhibit lower flow resistance. In the next step of our analysis, we enhance the bin width to $30^{\circ}$ and focus on two directions of preferential neurite growth: perpendicular to the acoustic propagation axis at $\alpha_{\perp}=\left(135^{\circ} \mp 15^{\circ}, 315^{\circ} \mp 15^{\circ}\right)$ and parallel to it at $\alpha_{\|}=\left(45^{\circ} \mp 15^{\circ}, 225^{\circ} \mp 15^{\circ}\right)$.

a

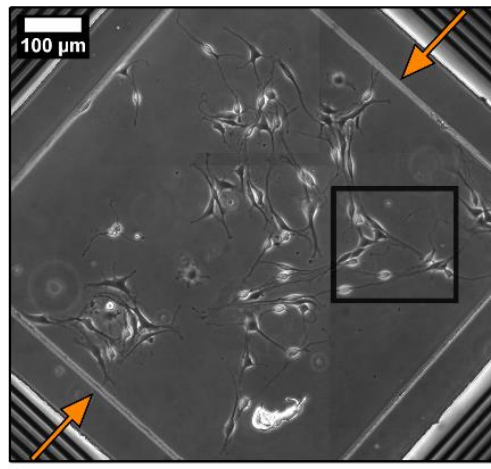

$\mathrm{PH}$ Image (B35 cells)

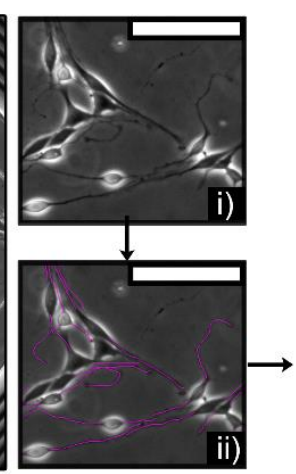

NeuronJ

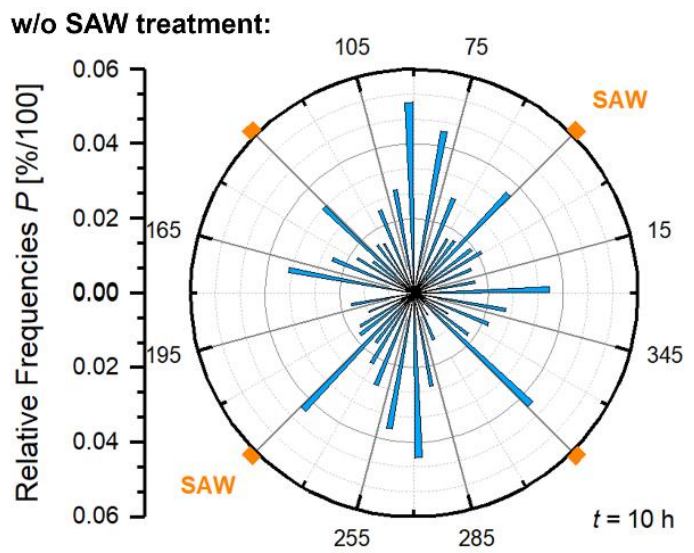

b $12 \mathrm{dBm} / \mathrm{PWM}=100 \%$ :

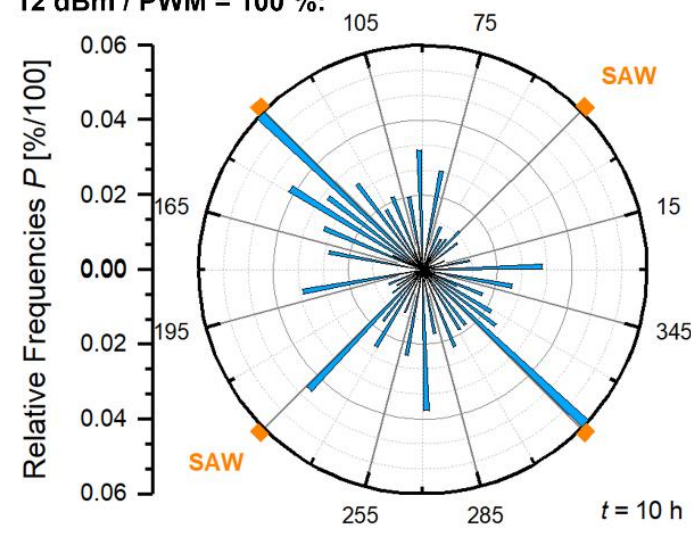

C $21 \mathrm{dBm} / \mathrm{PWM}=\mathbf{3 0} \%$ :

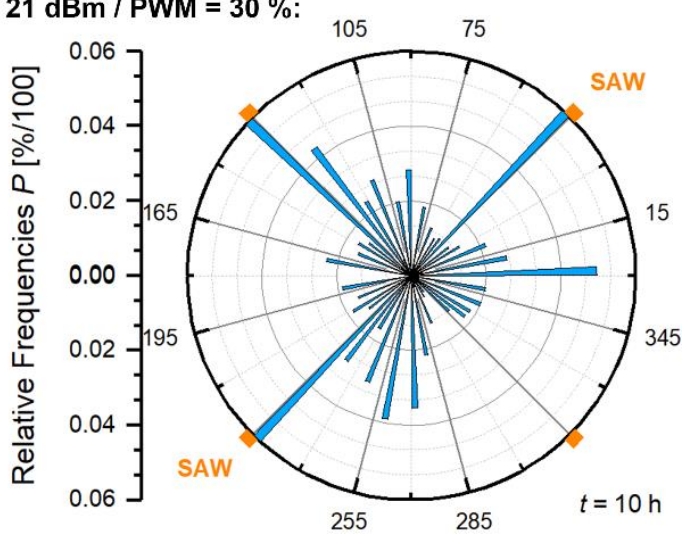

Figure 2: Exemplary neurite angle distributions after $10 \mathrm{~h}$ of SSAW treatment at different intensities and PWM. a) PH image of B35 cells in a microchannel and the corresponding angular distribution of neurites tracked by NeuronJ in a reference sample, where no SSAW was applied. The propagation direction of a ID SSAW (that is applied to SSAW-treated samples) is marked in orange. b) Neurite angular distribution in a SSAW-treated sample at $P_{\text {in }}=12 \mathrm{dBm}$ and $P W M=100 \%$. c) Distribution in a SSAW-treated sample at $P_{\text {in }}=21 \mathrm{dBm}$ and $P W M=30 \%$.

In Figure 3 a, we present a summary of the relative frequencies $f\left(\alpha_{\perp}\right)$ and $f\left(\alpha_{\|}\right)$from all samples as function of the mean acoustic force $\bar{F}=F_{\mathrm{R}}\left(P_{\mathrm{in}}\right) * \frac{\mathrm{PWM}}{100}$ at $t=10 \mathrm{~h}$ after the start of the SSAW treatment. The straight black line represents the respective mean frequency and standard deviation of three reference samples where no SSAW was employed. A percentage of $f\left(\alpha_{\perp}\right)=83.3 \%$ of the SSAW-treated samples lies clearly above the error range of the reference samples. We can therefore 
conclude that 1) the treatment with SSAW seems to promote neurite orientation along the nodes of the SSAW after $t=10 \mathrm{~h}$. However, no such effect becomes visible in the $\alpha_{\|}$direction. In total, the data in Figure 3 a are distributed between $f_{\min }=0.107$ and $f_{\max }=0.253$, but do not follow any visible forcedependent trend. We obtained similar results for $t>10 \mathrm{~h}$ (see SI 2a-c for the corresponding graphs at $t=20 \mathrm{~h}, 30 \mathrm{~h}$ and $40 \mathrm{~h}$ ). Therefore, the second conclusion that we can draw is that 2) neurite orientation is not dependent on the magnitude of the mean acoustic forces $\bar{F}$ in the here employed range. This allows us to summarize the data points by calculating the mean relative frequencies $\bar{P}\left(\alpha_{\perp}, t\right)$ and $\bar{P}\left(\alpha_{\|}, t\right)$ and their standard deviations $\Delta \bar{P}\left(\alpha_{\perp}, t\right)$ and $\Delta \bar{P}\left(\alpha_{\|}, t\right)$ from all the data points, as depicted in Figure $\mathbf{3} \mathrm{b}$ and $\mathrm{c}$. We then performed a two-sample $\mathrm{t}$ test to compare the SSAW-treated samples to the respective reference samples at each time step at a significance level of $\alpha=0.05$. The resulting $p$ value is included next to the SSAW mean values marked green if $p \leq \alpha$ (a table containing all calculated $t$ and corresponding $p$ values as well as the number of degrees of freedom $d f$ can be found in SI 3 ). For all mean values with $p \leq \alpha$, we can accept the null hypothesis stated the beginning of this section: We can confirm that $10 \mathrm{~h}$ of SSAW treatment can significantly $(p=0.043)$ promote neurite outgrowth along the pressure minima by up to $\frac{\bar{P}_{\mathrm{SAW}}\left(\alpha_{\perp}\right)-\bar{P}_{\text {Ref }}\left(\alpha_{\perp}\right)}{\bar{P}_{\text {Ref }}\left(\alpha_{\perp}\right)} \approx 23.5 \%$. For longer treatments $t>10 \mathrm{~h}$, this effect vanishes and even becomes reversed: At $t=30 \mathrm{~h}$, the treatment leads to a preferential neurite outgrowth parallel to the propagation direction of the SSAW by up to $\frac{\bar{P}_{\text {SAW }}\left(\alpha_{\|,}, 30 \mathrm{~h}\right)-\bar{P}_{\text {Ref }}\left(\alpha_{\|,}, 30 \mathrm{~h}\right)}{\bar{P}_{\operatorname{Ref}}\left(\alpha_{\|}, 30 \mathrm{~h}\right)} \approx$ $31.3 \%(p=0.029)$. As mentioned above, we ascribe the reverse effect to the acoustic streaming in the microchannel that outweighs the acoustic force in long-term SSAW treatments. According to our data, however, the acoustic force remains the main driving force for SSAW-directed neurite outgrowth for treatment durations up to $t=10 \mathrm{~h}$.

After $t=40 \mathrm{~h}$ of treatment, our results show no significant preference in any of the two directions. At the same time, we observed a drastic decline in the relative detected number of neurite segments $N$ in several samples, as visualized in Figure $3 \mathrm{~d}$. While after $t=20 \mathrm{~h}, 75 \%$ of all samples maintained the same or exhibited an even higher number of neurite segments, the segment numbers in some samples began to drop between $t=30$ to $40 \mathrm{~h}$. Although we cannot draw a clear conclusion about the correlation between $N$ and the mean acoustic force $\bar{F}$ from Figure $3 \mathrm{~d}$, the neurite shrinkage might be a sign of reduced cell viability. Our experiments indicate that high-intensity treatments often evoke early cell death after less than $t=20 \mathrm{~h}$, concealing any possible neurite outgrowth effects (the purple curve in Figure $3 \mathrm{~d}$ represents the only sample with cells surviving $20 \mathrm{~h}$ of treatment at $P_{\text {in }}=21 \mathrm{dBm}$ and PWM $=30 \%$ ). To discuss these effects and further explore the limits of applicable SSAW parameters for directed neurite outgrowth experiments, we provide a detailed analysis on cell viability in the following section. 
a

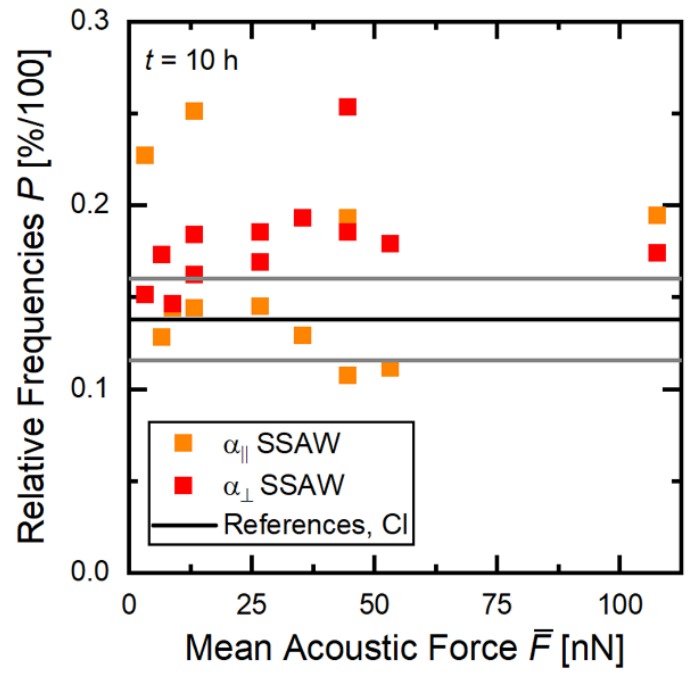

c

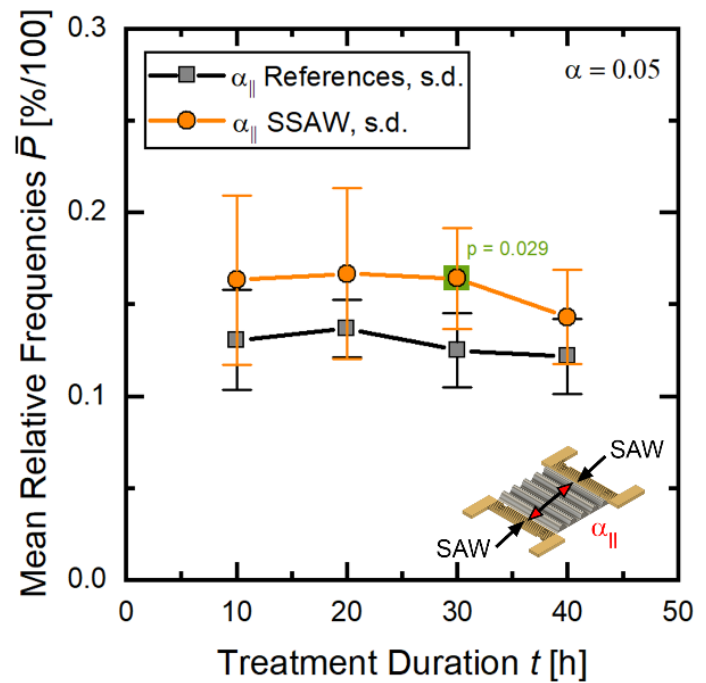

b

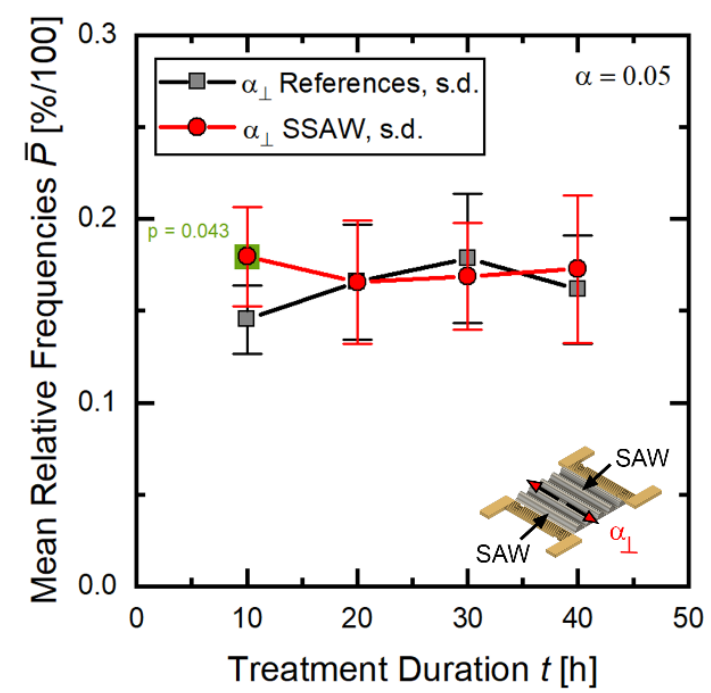

d

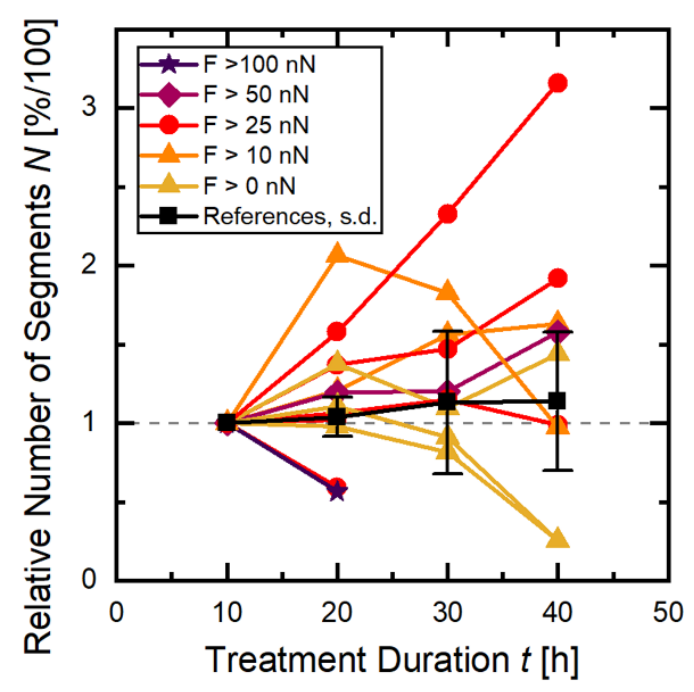

Figure 3: Overall results of SSAW-guided neurite outgrowth, summarized as relative frequencies of neurites growing in the directions perpendicular $\left(\alpha_{\perp}\right)$ and parallel $\left(\alpha_{\|}\right)$to the SSAW propagation direction, including standard deviations (s.d.). a) Exemplary distribution of the neurites after $t=10 \mathrm{~h}$ of SSAW treatment as a function of different PWM and power levels $P_{i n}$, summarized here as mean acoustic force $\bar{F}=F_{R}\left(P_{i n}\right) * P W M / 100$ and confidence interval $(C I)$. b) Mean relative frequencies of neurites growing in the $\alpha_{\perp}$ direction as a function of treatment duration $t$. A two-sample t test was performed to test the significance of the differences between SSAW-treated samples and reference samples. c) Mean relative frequencies of neurites growing in the $\alpha_{\|}$direction (analogous to b). d) Relative development of the number of neurite segments $N$ (detected by NeuronJ) in all samples over time.

\subsection{Effects of SSAW on apoptosis and necrosis}

To study cell viability, we prepared four samples from the same passage for each experiment, as described in section 5.1. One chip served as static reference with no SSAW employed. The other three chips were treated with a 1D standing SAW with pulse width modulations PWM $=30 \%, 50 \%$ and 100 $\%$ at a frequency of $1 \mathrm{~Hz}$ (in the case of PWM $=30 \%$, the signal is turned on for $0.3 \mathrm{~s}$ and turned off for $0.7 \mathrm{~s})$. 
We determined the percentage of apoptotic and necrotic cells in each time frame employing the machine learning software ilastik ${ }^{23}$ and the Matlab program Intensity Plotter ${ }^{20}$. An exemplary scheme showing the different steps of image analysis for necrotic cells is illustrated in Figure $\mathbf{4}$ a. The Intensity Plotter software detects the coordinates of the nuclei in the DAPI channel and determines the corresponding mean intensity value in the TexasRed channel (or in the FITC channel in case of apoptotic cells). This intensity is directly linked to the amount of propidium iodide bound to the nucleus and therefore serves as a measure for the cell's stage of necrosis. We chose an intensity threshold $I_{T}$ above which a cell is defined as necrotic and determined the proportion of necrotic cells $R$ in a sample:

$$
R(t)=\frac{N_{I>I_{T}}(t)}{N_{t o t}} .
$$

The fraction of apoptotic and necrotic cells $R(t)$ in the sample as a function of time follows a sigmoidal trend and can be approximated with a dose response curve, following the equation:

$$
y(t)=A_{a}+\frac{A_{a}-A_{e}}{1+10^{(w-t) p}},
$$

with $A_{\mathrm{a}}$ and $A_{\mathrm{e}}$ as the lower and upper asymptote, $w$ the turning point and $p$ the slope in this point. In the following, the turning point is referred to as $t_{\mathrm{LD} 50}$, i.e., the time when the SSAW dose is lethal to half of the cells in the sample.

We observed that the distribution of the time difference between necrosis and apoptosis is very sharp around $t_{\mathrm{LD} 50}=17.4+-1.1 \mathrm{~min}$ and that apoptosis signals occur shortly before the necrosis signals (see SI 4a). This indicates a consecutive secondary necrosis ${ }^{24}$ due to the lack of phagocytes in our in vitro setup, which would usually remove apoptotic cells in in vivo systems. The remaining apoptotic cells experience membrane damage after the time $\Delta t_{\mathrm{LD} 50}$, enabling propidium iodide to bind to the nucleus. In the following, we decided to only analyse necrosis because the detection of apoptosis does not yield any significant additional information.

In Figure $4 \mathrm{~b}$, we display exemplary plots and a fit curve of $R$, as determined by (4) and (5), in two samples treated with SSAW at $P_{\text {in }}=15 \mathrm{dBm}$, but with different pulse width modulations. In the sample treated with an SSAW signal at PWM $=30 \%$, the turning point $t_{\mathrm{LD} 50}$ is reached earlier than in the sample exposed to a continuous SSAW signal (PWM $=100 \%$ ). In the static reference, necrosis does not significantly increase during the whole experiment. We draw two conclusions from this graph: 1) The treatment of B35 cells with SSAW at $P_{\text {in }}=15 \mathrm{dBm}$ for several hours already affects cell viability. 2) The negative effect increases with increasing pulse width (which is proportional to the acoustic energy input).

To exclude day to day fluctuations of the cell culture's viability, we evaluated all samples relatively to the reference samples from the respective day of the experiment (for absolute $t_{\text {LD50 }}$ times see SI $\mathbf{4 b}$ ). This leads to the results shown in Figure $\mathbf{4} \mathrm{c}$, where we depict the correlation of the relative time $t_{\mathrm{LD} 50, \text { rel }}$ $=t_{\mathrm{LD} 50} / t_{\text {Ref }}$ as a function of increasing PWM for all employed SSAW power levels (per definition we obtain $t_{\mathrm{LD} 50, \text { rel }}=1.0$ for PWM $=0 \%$, because here $\left.t_{\text {Ref }}=t_{\mathrm{LD} 50}\right)$. For power levels $P_{\text {in }} \leq 12 \mathrm{dBm}$, we can conclude that the SSAW treatment does not reduce cell viability. We even see a small positive effect at $P_{\text {in }}=12$ and $4 \mathrm{dBm}$ with a maximum of $t_{\mathrm{LD} 50, \text { rel }}:=1.30$ at $\left(P_{\text {in }}{ }^{*} \mathrm{PWM}\right)=\left(12 \mathrm{dBm}{ }^{*} 50 \%\right)$. For higher power levels, this effect becomes reversed: $P_{\text {in }}=15 \mathrm{dBm}$ marks the limit above which SSAW started to affect the viability of our samples. For $P_{\text {in }}=15 \mathrm{dBm}$ and $18 \mathrm{dBm}$, the viability decreases almost linearly with increasing PWM, whereas at $21 \mathrm{dBm}$, the lethal dose is reached faster at $t_{\mathrm{LD} 50}(21 \mathrm{dBm}, 30 \%)=$ $0.34 * t_{\text {Ref. }}$. Hence, for $P_{\text {in }} \geq 15 \mathrm{dBm}$, the cells on SSAW-treated samples become necrotic significantly faster than the cells in our static reference. Consequently, the mean acoustic force $\bar{F}$, which was introduced in the previous section and combines the influence of both power level and PWM, is an important driving factor of SSAW-induced cell death in our sample (see SI 4c). 

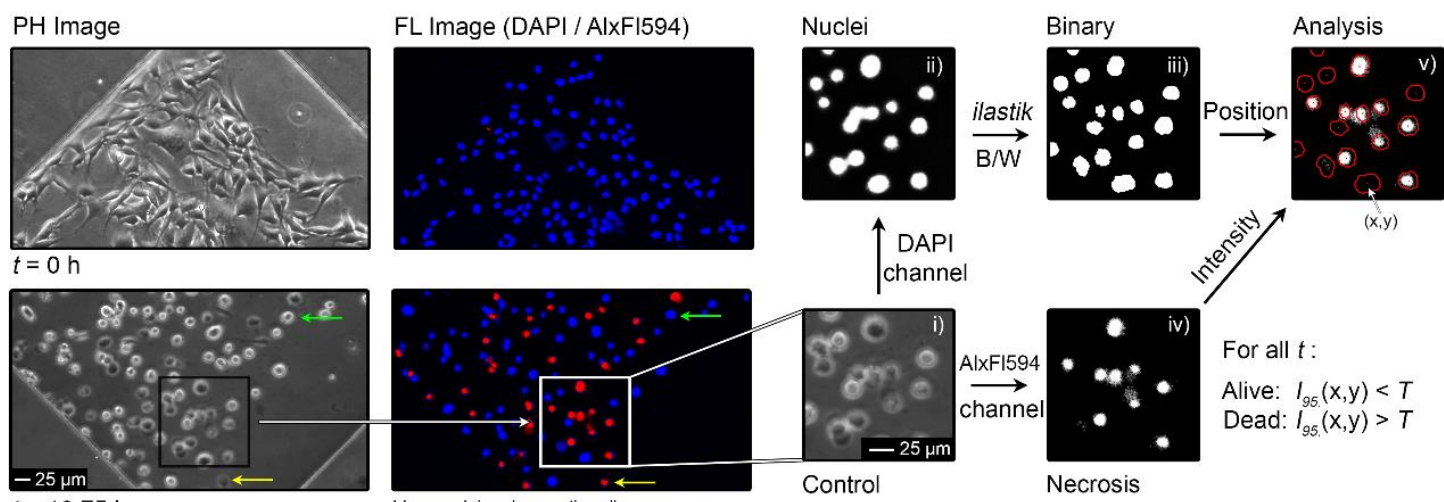

$\uparrow \begin{gathered}\text { DAPI } \\ \text { channel }\end{gathered}$
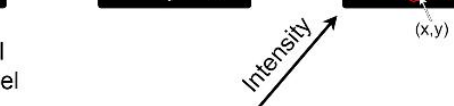

$t=16.75 \mathrm{~h}$

blue: nuclei, red: necrotic cells
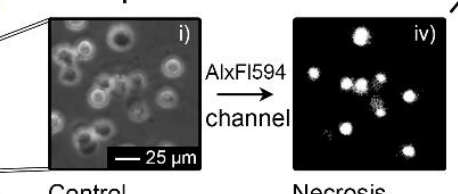

For all $t$ :

Alive: $I_{95}(\mathrm{x}, \mathrm{y})<T$

Dead: $I_{95}(\mathrm{x}, \mathrm{y})>T$

b

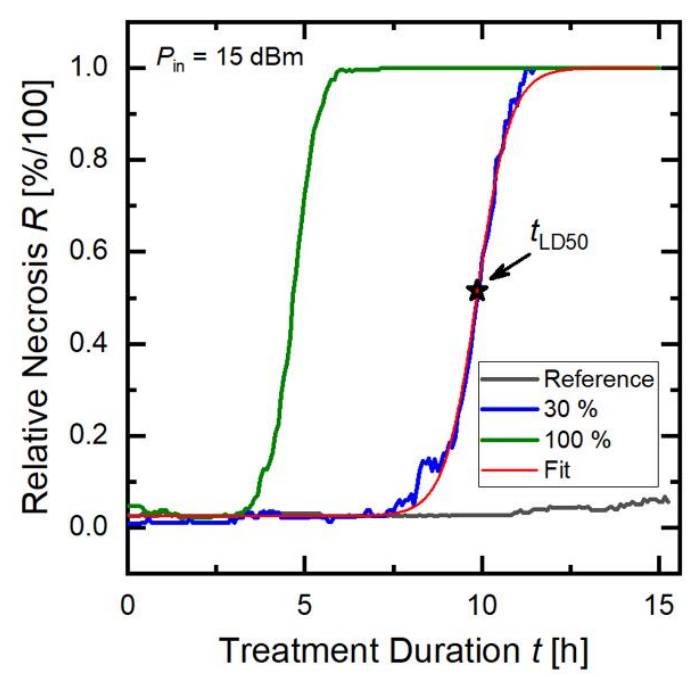

C

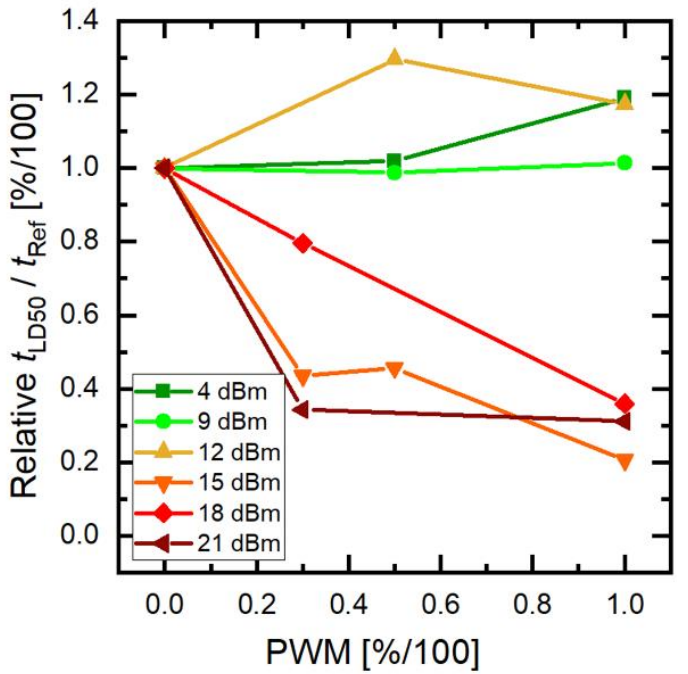

Figure 4: Results of B35 cell viability with SSAW treatment at different power levels $P_{\text {in }}$ and PWM. a) Analysis scheme and exemplary $\mathrm{PH}$ and fluorescence images of SSAW-induced necrosis. b) Relative necrosis in samples treated with different pulse width modulations at $P_{\text {in }}=15 \mathrm{dBm}$. c) Relative $t_{L D 50}$ times (relative to the reference) for different power levels as function of applied PWM (adapted from $\left.{ }^{20}\right)$.

To maintain cell viability in long-term SSAW experiments, it is therefore highly recommendable to employ low SSAW power levels up to $P_{\text {in }}=12 \mathrm{dBm}\left(\Delta \mathrm{T}_{\max }(12 \mathrm{dBm}, 50 \%)=1.0 \mathrm{~K}\right.$ for a 2D SSAW, according to Figure $1 \mathrm{c}$ ). The results show that, in our setup, high power levels are only applicable for a few hours. In setups containing active cooling or different geometries it might be possible to extend the applicable power levels by minimizing the thermal energy input on the active area of the chip, as we will discuss in detail in the next section. In this context, pulse width modulations between PWM = $30 \%$ and $50 \%$ should be chosen over a continuous SSAW signal to best maintain cell viability at power levels $P_{\text {in }} \geq 15 \mathrm{dBm}$. This is in accordance with our findings from section 2.2 where we showed that pulsed SSAW signals can also effectively direct neurite outgrowth.

To complete the picture of cell viability in our setup, we will analyse cell proliferation under SSAW influence in the next section. 


\subsection{Effects of SSAW on proliferation}

Based on our findings from the previous section, we decided to employ mild SSAW parameters in our proliferation assays to avoid early apoptosis and necrosis. Therefore, we here investigate a power range from $P_{\text {in }}=3-18 \mathrm{dBm}$, where all samples were treated with a 1D SSAW of PWM $=30 \%$ for $t=15$ h. In analogy to the data analysis of necrosis, the proportion of proliferated cells in each sample was determined employing ilastik and the Intensity Plotter software. Here, all cells with a mean intensity above an intensity threshold $I_{T}=0.3 * I_{\max }$ in the FITC channel are defined as proliferated, as shown in Figure 5 a.

a

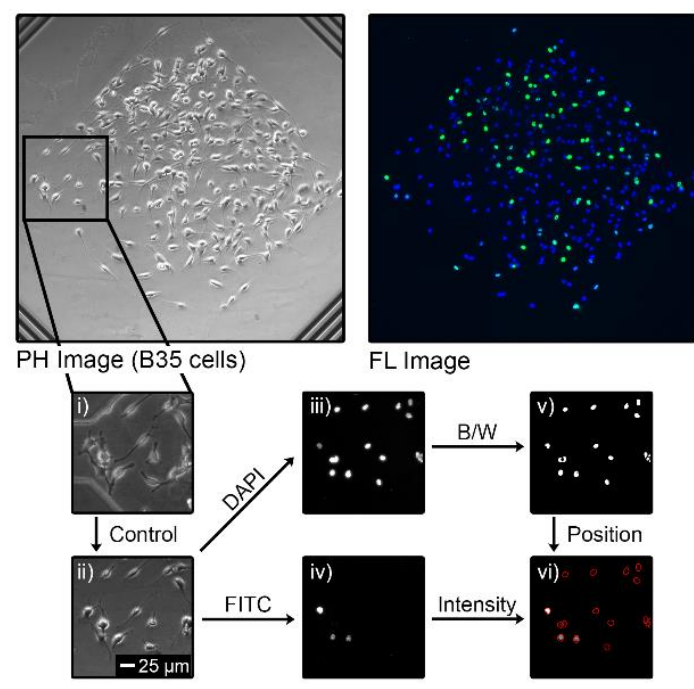

b

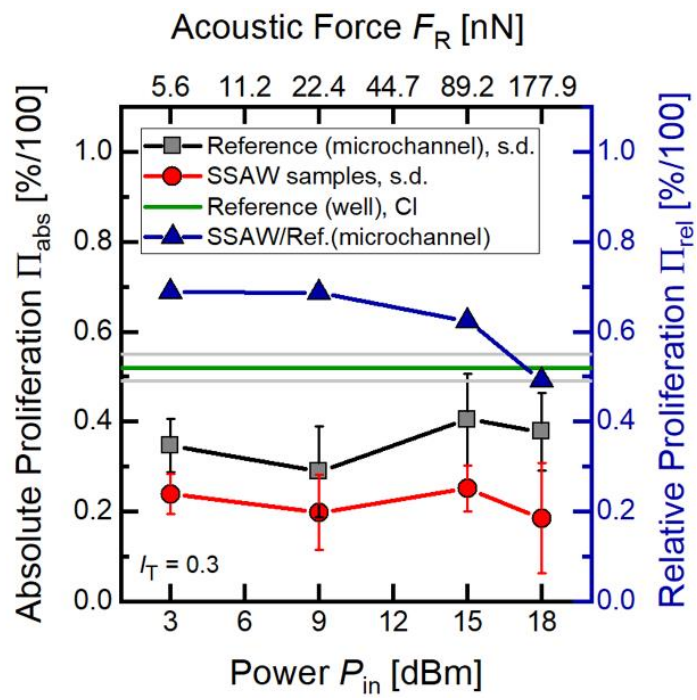

Figure 5: SSAW-influenced proliferation of B35 cells. a) Scheme and exemplary PH and fluorescent images of the proliferation analysis. b) Mean percentage of proliferated cells in samples treated with SSAW at PWM $=30 \%$ and in reference samples as function of applied power $P_{\text {in, }}$ including standard deviations (s.d.) and the confidence interval (CI) of the reference. Each power level corresponds to one set of experiments, where three SSAW samples and three reference samples (in microchannels) were measured.

As shown in Figure $5 \mathrm{~b}$, surprisingly the average proliferation on nearly all SSAW-treated samples was reduced in comparison to the static references. In contrast, in earlier studies an increase of proliferation during application of travelling SAW ${ }^{25}$ was found. At the highest power $P_{\text {in }}=18 \mathrm{dBm}$, proliferation $\Pi_{a b s}$ in the SSAW sample decreased even by up to $51 \%$ in comparison to the reference samples. However, the graph in Figure $5 \mathrm{~b}$ shows that low and medium powers of $P_{\text {in }}=3 \mathrm{dBm}$ and 15 $\mathrm{dBm}$ affected cell division behaviour as well. The reference samples were prepared at the same day as the corresponding SSAW samples but were not treated with SSAW (in Figure $\mathbf{5}$ b, each power level corresponds to one set of experiments). For a better visualization, we determined the average relative proliferation $\Pi_{\text {rel, }}$ shown as blue graph. Here, $\Pi_{\text {rel }}<1.0$ for all SSAW power levels. This fact and the single proliferation values of each sample (see SI 5 a) confirm that reduced proliferation appeared in almost all SSAW-treated samples. As we describe in the next section in detail, temperature effects do not play a role at powers $P_{\text {in }} \leq 15 \mathrm{dBm}$ : The maximum temperature increase for $P_{\text {in }}=15 \mathrm{dBm}$ and PWM $=30 \%$ in the sample is only $\Delta \mathrm{T}=0.78 \mathrm{~K}$ (Figure $1 \mathrm{c}$ ), so that temperature effects are most likely not responsible for reduced proliferation at power levels $P_{\text {in }} \leq 15 \mathrm{dBm}$.

Interestingly, we also observed different proliferation behaviour between samples with and without microchannels. The latter is included in Figure $\mathbf{5} \mathrm{b}$ as a green line and consists of an unrestricted external reference, where we seeded cells in a 4-well culture dish and determined the mean proliferation and confidence interval after $t=15 \mathrm{~h}$ of incubation at $37{ }^{\circ} \mathrm{C}$ and $5 \% \mathrm{CO}_{2}$. The proliferation 
outside of our setup was overall higher than inside the height-restricted microchannels. We ascribe this effect to the higher volume $\left(V_{\text {well }}=1 \mathrm{ml}\right)$ of medium in the wells than in the channels $\left(V_{\text {channel }}=\right.$ $0.013 \mu \mathrm{l})$, which leads to an overall better distribution of nutrients among the cells.

We can conclude that, in addition to the geometry-induced effects in the microchannel, proliferation in our setup is reduced by the SSAW treatment. Moreover, this negative effect increases with higher SSAW intensity.

\section{DISCUSSION}

In the following, we describe and evaluate three main factors that could contribute to the effects on cell viability from section 2.2 and 2.3. These are i) cell density, ii) temperature effects, and iii) acoustic forces. This discussion finally leads us to the conclusion that the effects most likely originate from acoustic forces (for all power levels $P_{\text {in }} \leq 18 \mathrm{dBm}$ ), and additionally from increased temperature (for effects at power levels $P_{\text {in }} \geq 18 \mathrm{dBm}$ ).

\subsection{Cell density}

From literature it is well known that proliferation is sensitive to cell density. Puliafito et al. showed that starting from a critical area of $A_{\text {crit }}=200 \mu \mathrm{m}^{2}$ contact inhibition becomes relevant and leads to reduced proliferation of MDCK-II cells ${ }^{26}$. We additionally considered the correlation between proliferation and cell density of all samples to exclude this potential factor not related with the SSAW itself (see SI $\mathbf{5 b}$ ). The linear fit includes only the static reference samples and shows a slope of the order $10^{-4} \mathrm{~mm}^{-2}$, that is mainly determinated by only two samples of extremely high density. This study demonstrates that cell densities up to about $1500 \mathrm{~mm}^{-2}$ do not affect the cell division behaviour. Consequently, samples with higher cell densities were excluded in our statistics. We therefore ascribe the main effects that lead to reduced proliferation in Figure $\mathbf{5} \mathrm{b}$ to SSAW-related quantities, as we describe in the following.

\subsection{Temperature effects}

In section 2.1, we already discussed the heating of the substrate that results from high SSAW powers. As shown in Figure $1 \mathrm{c}$, low pulse width modulations induce smaller temperature changes than high PWM: At $P_{\text {in }}=15 \mathrm{dBm}$, the increase for the $50 \%$ pulsed 2D SSAW is $\Delta \mathrm{T}(15 \mathrm{dBm}, 50 \%)=2.56 \mathrm{~K}$, while the $30 \%$ pulsed 1D SSAW induces a temperature difference of only $\Delta \mathrm{T}(15 \mathrm{dBm}, 30 \%)=0.78 \mathrm{~K}$. The latter value lies below physiological afebrile temperature fluctuations of $\Delta \mathrm{T}=\mp 1 \mathrm{~K}$ and therefore should not affect cell viability. At $P_{\text {in }}=18 \mathrm{dBm}$, however, we observed elevated temperatures of $T>38$ ${ }^{\circ} \mathrm{C}$. For even higher power levels $P_{\text {in }}>21 \mathrm{dBm}$, critical temperatures of $T>40{ }^{\circ} \mathrm{C}$ were reached. This temperature limit is known to induce a strong inhibition of cell viability and proliferation ${ }^{27,28}$. The drastic changes in temperature for high power levels $P_{\text {in }} \geq 18 \mathrm{dBm}$ explain the decline of the relative proliferation curve in Figure $\mathbf{5} \mathrm{b}$ and the increase of apoptotic and necrotic cells at high SSAW powers and high PWM in Figure $4 \mathrm{c}$. Still, temperature cannot be the major driving force behind effects that we observed for $P_{\text {in }} \leq 15 \mathrm{dBm}$.

As mentioned above, different geometries like a small active area $A$ between the bidirectional IDTs can lead to high power densities in the area. The maximum power density $\Phi$ that we employed in our

setup for the proliferation experiments at $P_{\text {in }}=18 \mathrm{dBm}$ (i.e. $64 \mathrm{~mW}$ ) is $\Phi\left(P_{\mathrm{in}}, \mathrm{PWM}, A\right)=P_{\text {in }} \frac{P W M}{100 \mathrm{~A}}=64$ $\mathrm{mW} * 0.3 / 0.36 \mathrm{~mm}^{2} \approx 53.3 \mathrm{~mW} / \mathrm{mm}^{2}$. This energy density (and thus the heating of the substrate) in future experiments can be reduced by choosing a larger active area, which will shift the maximum of applicable SSAW power towards higher levels. 


\subsection{Acoustic forces}

For powers $P_{\text {in }} \leq 15 \mathrm{dBm}$, we observed that proliferation in SSAW-treated samples is still reduced by up to $37.7 \%$ relative to the external microchannel reference. Acoustic forces could be an explanation for this effect: Cells in acoustic potential landscapes are exposed to acoustic pressure and acoustic forces.

In section 2.1, we determined the maximum acoustic force acting on cells in our setup using equation (1). The forces corresponding to the employed SSAW power levels are shown in Figure $\mathbf{5}$ b on the upper $x$ axis (the values are only exact for cells in suspension and can vary slightly for adherent cells). In our experiments, the minimum and maximum acoustic force applied to the cells were $F_{\mathrm{R}, \min }(3 \mathrm{dBm})=5.6$ $\mathrm{nN}, F_{\mathrm{R}, \max }(18 \mathrm{dBm})=177.9 \mathrm{nN}$ and $F_{\mathrm{R}, \max }(21 \mathrm{dBm})=359.2 \mathrm{nN}$. Our results indicate that low forces of about $6 \mathrm{nN}$ already affect cell proliferation in this setup. Interestingly, Cattin et al. (2015), reported that mitosis behaviour of HeLa cells can be accelerated by low mechanical pressure $(F=5 \mathrm{nN})^{8}$. This is contrary to our observation and indicates that pulsed lateral acoustic forces might have different effects on proliferation behaviour than continuous transversal forces, as exerted by an AFM cantilever in Cattin et al.. A possible explanation for this deviation could be that vertical deformation induce different cellular reactions than lateral forces. This might be due to the anisotropy of the cell, like the geometry of the microtubule spindle apparatus which is known to play an important role in mitosis ${ }^{29}$. For higher forces between $F=50-100 \mathrm{nN}$, Cattin et al. observed a deceleration of cell division, leading up to an entire inhibition of proliferation at $F>100 \mathrm{nN}$. This is in accordance with our findings: At $P_{\text {in }}=$ $15 \mathrm{dBm}$, the cells were exposed to forces of $F_{\mathrm{R}}=89.2 \mathrm{nN}$, which impaired cell viability and led to reduced cell proliferation even at a pulse width modulation of only $30 \%$. Additional findings in literature indicate that the duration of incubation in the acoustic potential landscape is another crucial factor: Hultström et al. (2007) reported that neither proliferation nor viability of cells in a standing surface acoustic wave field were impaired during short SSAW treatments between $t=30-75 \mathrm{~min}^{7}$. Our experimental parameters exceed this duration by far, which could amplify the negative effects.

These considerations provide strong evidence that during long-term SSAW treatments of $t \geq 15 \mathrm{~h}$, cell proliferation in a microchannel setup on the chip is reduced because of acoustic forces. However, cell viability is not impaired at low forces (power levels $P_{\text {in }} \leq 12 \mathrm{dBm}$ ).

\section{CONCLUSION}

Our experiments reveal that long-term maintenance of cell viability in this setup, especially at high SSAW power levels, remains challenging. As cell proliferation is reduced even at low power and pulsed signals, it is extremely advisable to control environmental parameters such as temperature, $\mathrm{CO}_{2}$ saturation and supply with nutrients as carefully as possible. However, we found that during shortterm measurements up to $t=10 \mathrm{~h}$, neurites indeed orientate along the acoustic pressure nodes. Our data show that pulsed as well as continuous SSAW signals yield promising results in the field of SSAWdirected neurite growth and, at the same time, maintain cell viability long enough to observe statistically significant effects.

In their pioneering work, Brugger et al. (2018) observed a correlation of primary neurite outgrowth with an employed 2D acoustic potential landscape in a microchannel on SSAW chips. The behaviour of the B35 cell line can deviate from primary neurons in vivo; nevertheless, we were able to corroborate and extend these previous observations by studying the long-term effects of SSAW fields on cell 
viability, proliferation and guided neurite outgrowth at different power levels. We defined the limits of applicable SSAW power levels and found that pulsed SSAW signals enable higher intensity treatments up to several hours. Based on these parameters, we were able to significantly improve the investigations on SSAW directed neurite outgrowth reported earlier and demonstrated that B35 neurite orientation can be influenced using acoustic potential landscapes at pulsed high SSAW power levels. In future experiments, it would be illuminating to investigate neurite outgrowth employing shorter wavelengths, constricting neurite growth to narrower potential valleys. Additionally, microchannels with a smaller height could help to further suppress effects due to acoustic streaming. Another future extension could additionally include soft environments, such as polyacrylamide gels with adjustable Young's modulus or collagen hydrogels ${ }^{30}$. Application to intact tissue like, e.g. retina, would be fascinating. We expect that our findings will improve the efficiency of future research in this field and further pave the road towards the acoustic manipulation of neuron outgrowth and signalling.

\section{MATERIALS AND METHODS}

\subsection{Experimental setup}

For all experiments presented here, we cultivated B35 neuroblastoma cells in Dulbecco's Modified Eagle's Medium (DMEM, 4 mM L-glutamine, $4500 \mathrm{mg} / \mathrm{l}$ glucose, $1 \mathrm{mM}$ sodium pyruvate, $1500 \mathrm{mg} / \mathrm{l}$ $\mathrm{NaHCO}_{3}$ by ATCC) containing $10 \%$ fetal bovine serum (FBS Superior stabil ${ }^{\circledR}$ by Bio\&Sell) at $37^{\circ} \mathrm{C}$ and 5 $\% \mathrm{CO}_{2}$. The SAW chip consists of a $\mathrm{SiO}_{2}$-covered $\mathrm{LiNbO}_{3}$ substrate with four interdigital transducers (IDTs) made of thin metal layers (Ti/Au/Ti, 10/50/10 nm). The wavelength of the $\mathrm{SAW}$ is dependent on the transducer design. We chose $\lambda=50 \mu \mathrm{m}(f=71 \mathrm{MHz})$, because this is in the size range of a living cell. For single object SAW patterning using polystyrene microbeads, the diameter $D$ of the object should be in the range of $3.2 \geq \lambda / D \geq 3.6$, according to previous works by Collins et al. $(2015)^{31}$.

The active area of the chip was incubated with a Poly-L-Lysine (PLL, $0.1 \% \mathrm{w} / \mathrm{v}$ by Sigma Aldrich) solution containing $0.4 \% \mathrm{PLL}$ in PBS at room temperature for $t=1 \mathrm{~h}$. To suppress acoustic streaming a PDMS microchannel $(h=37 \mp 1 \mu \mathrm{m})$ and a polystyrene cover slide were placed on top of the microchannel to stabilize the setup and to enable the diffusion of fresh medium from the $1 \mathrm{ml}$ well on top of the slide into the channel.

The B35 cell suspension was pumped into the channel at a flow rate of $Q=0.005 \mu \mathrm{l} / \mathrm{s}$ using a polytetrafluoroethylene (PTFE) tube, a $250 \mu$ l syringe (Hamilton Bonaduz AG, Switzerland) and a neMESYS syringe pump (Cetoni $\mathrm{GmbH}$, Korbussen, Germany). The cells were patterned in the nodes of the standing wave field by applying a 2D SSAW at a power of $P_{\text {in }}=18 \mathrm{dBm}$. For signal generation, a customized multifunctional frequency generator with a built-in network analyzer by $M$. Brugger ${ }^{20}$ was used. Prior to the experiments, the complete experimental setup containing the nutrient was preincubated at $37^{\circ} \mathrm{C}$ and $5 \% \mathrm{CO}_{2}$ for several hours to ensure stable conditions ( $\mathrm{pH}$ and temperature) as well as stable cell adhesion and spreading.

\subsection{Detection of apoptosis, necrosis and proliferation}

After pre-incubation, we added $30 \mu$ of the fluorescent CellEvent Caspase-3/7 Green ReadyProbes Reagent per $1 \mathrm{ml}$ well to detect apoptotic cells and the same volume of the Propidium lodide ReadyProbes Reagent to detect necrotic cells (both dyes were purchased from Invitrogen ${ }^{\mathrm{TM}}$ ThermoFisher Scientific, Massachusetts, USA). To keep track of the total number of cells in the sample during the experiment, a volume of $30 \mu \mathrm{l}$ of Hoechst 33342 (NucBlue Live ReadyProbes Reagent, Invitrogen ${ }^{\mathrm{TM}}$ ThermoFisher Scientific, Massachusetts, USA) was added to the medium. We 
performed life cell imaging experiments employing a 1D SSAW at different power levels and pulse widths in an ibidi Stage Top Incubation System (for Multi-Well Plates, K-Frame, $\mathrm{CO}_{2} / \mathrm{O}_{2}$ ) every $5 \mathrm{~min}$ for $t=15$ to $20 \mathrm{~h}$. The fluorescent images were taken with a Zeiss Axiovert 200M microscope (Zeiss AG, Oberkochen, Germany) and a Hamamatsu camera (Orca 5G, Hamamatsu Photonics Germany GmbH, Herrsching am Ammersee, Germany).

In separate experiments, we detected the proportion of proliferated cells in a sample. After cell patterning, the medium in the well was substituted with fresh medium containing $20 \mu \mathrm{M}$ EdU (5-ethyl$2^{\prime}$-deoxyuridine) from the Click-iT ${ }^{\mathrm{TM}}$ EdU Cell Proliferation Kit for Imaging (Invitrogen ${ }^{\mathrm{TM}}$ ThermoFisher Scientific, Massachusetts, USA). As for apoptosis and necrosis experiments, the samples were treated with a 1D SSAW of various power levels $\left(P_{\text {in }}=3,9,15\right.$ and $\left.18 \mathrm{dBm}\right)$, but exclusively at a pulse width modulation of PWM $=30 \%$ for $t=15 \mathrm{~h}$. As the labelling procedure requires the removal of the microchannel from the substrate after the experiment, phase contrast images of the sample before and after the removal were compared to ensure that no cells were accidentally detached from the chip surface. The nuclei of the fixed cells were marked with $30 \mu \mathrm{l}$ of Hoechst 33342 (NucBlue Live ReadyProbes Reagent, Invitrogen ${ }^{\mathrm{TM}}$ ThermoFisher Scientific, Massachusetts, USA). 


\section{REFERENCES}

M. S. Brugger, S. Grundeen, A. Doyle, L. Theogarajan, A. Wixforth and C. Westerhausen, Phys. Rev. E, 2018, 98, 12411.

D. Peng, W. Tong, D. J. Collins, M. Ibbotson, S. Prawer and M. E. Stamp, Front. Neurosci., 2021, $15,37$.

W. Zhou, J. Wang, K. Wang, B. Huang, L. Niu, F. Li, F. Cai, Y. Chen, X. Liu and X. Zhang, Lab Chip, 2017, 17, 1725-1731.

F. Guo, Z. Mao, Y. Chen, Z. Xie, J. P. Lata, P. Li, L. Ren, J. Liu, J. Yang and M. Dao, Proc. Natl. Acad. Sci. U. S. A., 2016, 113, 1522-1527.

J. Shi, D. Ahmed, X. Mao, S.-C. S. Lin, A. Lawit and T. J. Huang, Lab Chip, 2009, 9, 2890-2895. A. Ozcelik, J. Rufo, F. Guo, Y. Gu, P. Li, J. Lata and T. J. Huang, Nat. Methods, 2018, 15, 10211028.

J. Hultström, O. Manneberg, K. Dopf, H. M. Hertz, H. Brismar and M. Wiklund, Ultrasound Med. Biol., 2007, 33, 145-51.

C. J. Cattin, M. Düggelin, D. Martinez-Martin, C. Gerber, D. J. Müller and M. P. Stewart, Proc. Natl. Acad. Sci. U. S. A., 2015, 112, 11258-11263.

M. H. Hsieh and H. T. Nguyen, Int. Rev. Cytol., 2005, 245, 45-90.

L. A. Ambattu, A. Gelmi and L. Y. Yeo, Small, 2022, 2106823, 2106823.

G. S. Kino., Acoustic waves: devices, imaging, and analog signal processing, Englewood Cliffs, New Jersey, 1987.

K. Yosioka and Y. Kawasima, Acta Acust. united with Acust., 1955, 5, 167-173.

L. Gor'kov, Dokl. Akad. Nauk Sssr, 1961, 88-92.

E. Gerlach, P. Grosse and E. Gerstenhauer, in Physik-Übungen für Ingenieure, Vieweg+Teubner Verlag, Wiesbaden, 1995, pp. 69-70.

B. Laboratories, Material Properties of Polystyrene and Poly(methyl methacrylate) (PMMA) Microspheres, https://www.bangslabs.com.

D. Hartono, Y. Liu, P. L. Tan, X. Y. S. Then, L.-Y. L. Yung and K.-M. Lim, Lab Chip, 2011, 11, 4072-4080.

M. E. M. Stamp, M. S. Brugger, A. Wixforth and C. Westerhausen, Biomater. Sci., 2016, 4, 1092-1099.

R. Samy, T. Glawdel and C. L. Ren, Anal. Chem., 2008, 80, 369-375.

D. ROSS, M. Gaitan and Le Locascio, Anal. Chem., 2001, 73, 4117-4123.

M. S. Brugger, University of Augsburg, 2021.

E. Meijering, M. Jacob, J.--C. Sarria, P. Steiner, H. Hirling and M. Unser, Cytom. Part A J. Int. Soc. Anal. Cytol., 2004, 58, 167-176.

M. F. Schneider, Z. Guttenberg, S. W. Schneider, K. Sritharan, V. M. Myles, U. Pamukci and A. Wixforth, ChemPhysChem, 2008, 9, 641-645.

S. Berg, D. Kutra, T. Kroeger, C. N. Straehle, B. X. Kausler, C. Haubold, M. Schiegg, J. Ales, T. Beier and M. Rudy, Nat. Methods, 2019, 16, 1226-1232.

T. Vanden Berghe, N. Vanlangenakker, E. Parthoens, W. Deckers, M. Devos, N. Festjens, C. J. Guerin, U. T. Brunk, W. Declercq and P. Vandenabeele, Cell Death \{|\&\} Differ., 2010, 17, 922930.

M. S. Brugger, K. Baumgartner, S. C. F. Mauritz, S. C. Gerlach, F. Röder, C. Schlosser, R. Fluhrer, A. Wixforth and C. Westerhausen, Proc. Natl. Acad. Sci. U. S. A., 2020, 117, 31603-31613.

A. Puliafito, L. Hufnagel, P. Neveu, S. Streichan, A. Sigal, D. K. Fygenson and B. I. Shraiman, Proc. Natl. Acad. Sci. U. S. A., 2012, 109, 739-744.

H. Okumura, Y. Udagawa, K. Yamada, K. Tsukasaki, Y. Azuma and S. Nozawa, Proc. Japan Acad. Ser. B, 1979, 55, 135-140.

J. L. Roti Roti, Int. J. Hyperth., 2008, 24, 3-15.

P. Lara-Gonzalez, F. G. Westhorpe and S. S. Taylor, Curr. Biol., 2012, 22, R966-R980.

P. Delsing, A. N. Cleland, M. J. A. Schuetz, J. Knörzer, G. Giedke, J. I. Cirac, K. Srinivasan, M. Wu, K. C. Balram and C. Bäuerle, J. Phys. D. Appl. Phys., 2019, 52, 353001. 
31 D. J. Collins, B. Morahan, J. Garcia-Bustos, C. Doerig, M. Plebanski and A. Neild, Nat. Commun., 2015, 6, 1-11. 


\section{Supplementary Files}

This is a list of supplementary files associated with this preprint. Click to download.

- Supportinglnformation.pdf 